\title{
Numerical Simulation of the Plunger Velocity
}

\author{
K.Laevsky, R.M.M. Mattheij \\ Department of Mathematics and Computer Science, \\ Eindhoven University of Technology, \\ PO Box 513, 5000 MB The Netherlands
}

January 11, 2001

\section{Introduction}

A typical stage in the manufacturing of container glass, such as bottles and jars, is the pressing phase. At this stage a gob of hot glass ( $1000 \mathrm{C}$ or higher) is pressed in a mechanical construction by a so called plunger. This paper describes an important part of the simulation, the motion of the plunger. The problem is to determine the velocity of the latter which appears as a (kinematic) boundary condition of the glass flow problem. We show how to effectively uncouple these and to solve the resulting stiff differential equation numerically.

The flow can be described by the Stokes equations. For a more detailed description see [1]. We remark that the problem can be considered to be axisymmetric. Let us denote by $r, z$, and $\phi$ the radial, axial and azimuthal coordinates. The Stokes problem (in cylindrical coordinates) can then be formulated as follows. Find a velocity field $\mathbf{v}=\left(u_{r}(r, z), u_{z}(r, z), 0\right)^{T}$ and a pressure field $p=p(r, z)$, which satisfy

$$
\begin{aligned}
\operatorname{div} \sigma(\mathbf{v}, p) & =0 \\
\operatorname{div} \mathbf{v} & =0
\end{aligned}
$$

where $\sigma(\mathbf{v}, p)$ is a stress tensor.

As we have an axisymmetric problem we obtain a domain $\Omega$ as sketched in Figure 1 . The boundary $\Gamma:=\partial \Omega$ of domain consists of four parts

$$
\Gamma=\Gamma_{s} \cup \Gamma_{m} \cup \Gamma_{p} \cup \Gamma_{f},
$$

where the indices $s, m, p, f$ represent the symmetric, mould, plunger and free boundaries respectively, for which we define suitable boundary conditions.

As for the plunger which moves with velocity $\mathbf{v}_{p}$, we find

$$
\begin{aligned}
\left(\mathbf{v}-\mathbf{v}_{p}\right) \cdot \mathbf{n} & =0 \\
\left(\sigma \mathbf{n}+\beta_{p}\left(\mathbf{v}-\mathbf{v}_{p}\right)\right) \cdot \mathbf{t} & =0
\end{aligned}
$$




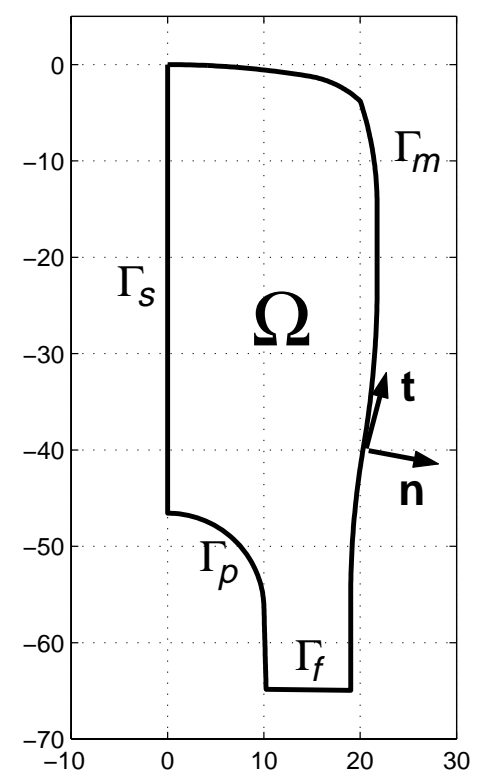

Figure 1.1: Problem domain.

Note that $\mathbf{v}_{p}$ does not depend on $r, z$, and $\beta_{p}$ is a friction coefficient. Let $V_{p}>0$ be the absolute velocity of the plunger, then

$$
\mathbf{v}_{p}=V_{p} \mathbf{e}_{z}=\left(0, V_{p}, 0\right)^{T}
$$

Actually, the velocity of the plunger $V_{p}$ is an unknown function of time $t$, so one should write $V_{p}(t)$. Nevertheless, for the boundary conditions below and the Stokes problem as such, we say this is just a parameter. Hence, the boundary conditions read as follows

$$
\begin{aligned}
\mathbf{v} \cdot \mathbf{n} & =V_{p} \mathbf{e}_{z} \cdot \mathbf{n}, \\
\left(\sigma \mathbf{n}+\beta_{p} \mathbf{v}\right) \cdot \mathbf{t} & =\beta_{p} V_{p} \mathbf{e}_{z} \cdot \mathbf{t} .
\end{aligned}
$$

One should note that the velocity field found from (1.1), (1.2) with the boundary conditions is independent of the value of $p_{0}$.

Once the boundary conditions are defined, (1.1), (1.2) can be solved numerically using a finite element approach, for example. As a result the velocity field $\mathbf{v}$ and the pressure $p$ can be obtained. One can see that equations (1.1), (1.2) are time independent. In order to find evolution in time we shall integrate our domain $\Omega_{t^{n}}$ at time $t^{n}$ using correspondent solution of Stokes equations. As a result we obtain $\Omega_{t^{n+1}}$ which is then used to define the Stokes equations and boundary conditions. These steps can be continued until the glass will fill the physical domain defined by the mould and the plunger geometries. This procedure is not described in this paper, for more detail see [1].

Clearly, in order to define (1.7), (1.8) we need the velocity of the plunger $V_{p}(t)$ to be known at time $t^{n}$. On the other hand, as it will be detailed in the next section, $V_{p}\left(t^{n}\right)$ depends on the solution of Stokes equations. 


\section{Plunger motion}

Consider now the velocity of the plunger $V_{p}(t)$, which was previously assumed to be a parameter in the boundary conditions. Let $F(t)$ denote the total force on a plunger and $m_{p}$ be the mass of the plunger. Then

$$
m_{p} a(t)=F(t), \quad a(t)=\frac{d V_{p}(t)}{d t}
$$

The total force is the sum of

$$
F(t)=F_{p}+F_{g}(t) .
$$

Here $F_{p}$ is the result of a constant pressure on the plunger, i.e.

$$
F_{p}=S_{p} p_{p}=\text { const }
$$

where $S_{p}$ is the area of the surface where pressure $p_{p}$ is applied. The second term $F_{g}(t)$, is the force on the plunger from the glass. It expressed in terms of the stress tensor (??)

$$
F_{g}(t)=\int_{S(t)} \sigma \mathbf{n} d S
$$

where $\sigma \equiv \sigma(t)$ is the stress tensor, and $S(t)$ is a part of the plunger surface which is in contact with the glass at time $t$.

Now, in order to compute the velocity of the plunger $V_{p}(t)$ as a function of time, one should solve the ordinary differential equation:

$$
\left\{\begin{aligned}
\frac{d V_{p}(t)}{d t} & =\frac{F_{g}(t)}{m_{p}}+\frac{F_{p}}{m_{p}}, \\
V_{p}(0) & =V_{p_{0}},
\end{aligned}\right.
$$

where $V_{p_{0}}$ is some initial velocity of the plunger. Note that we can compute $F_{g}(t)$, once $u_{r}, u_{z}$ and $p$ are known. The latter are obtained from the solution of Stokes equations (1.1), (1.2). In order to solve the Stokes equations one should define (1.7), (1.8) using some value for the plunger velocity $V_{p}$. So, at time $t=0$ we use $V_{p_{0}}$ from (2.2). Given this $F_{g}(t)$ we can perform an integration step in (2.2). Suppose we naively use the Euler Forward scheme

$$
V_{p}^{n+1}=V_{p}^{n}+\Delta t a^{n}, \quad a^{n}=a\left(t^{n}\right) .
$$

Having solved the Stokes equations, with the new velocity of the plunger $V_{p}^{n+1}$ we can complete the boundary conditions for the Stokes problem at $t=t^{n+1}$. For this purpose the velocity of the plunger obtained from (2.3) is used. This problem turns out to be stiff, as is illustrated in Figure 2.1. A fully implicit scheme is practically impossible, as we do not know the plunger velocity at $t^{n+1}$, and can not thus use it for the boundary conditions (1.8), (1.8). 


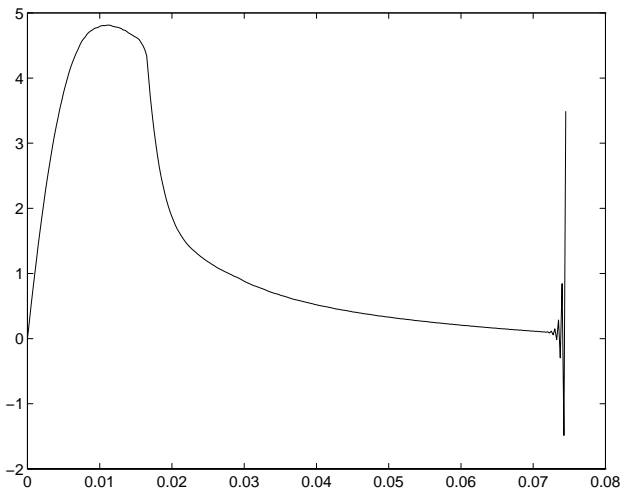

(a)

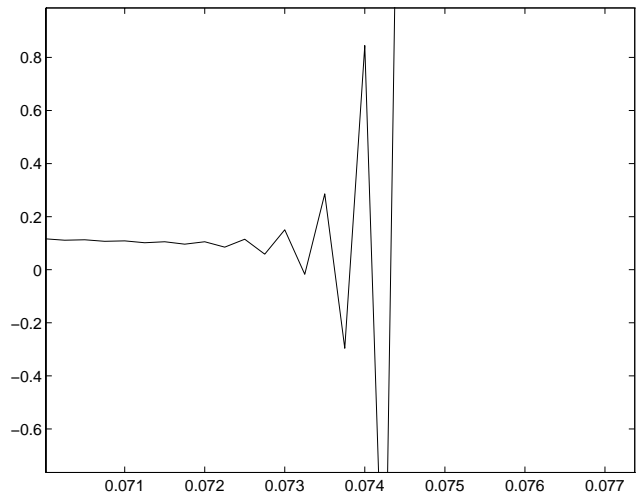

(b)

Figure 2.1: Velocity of the plunger (numerical instabilities).

\section{Uncoupling $V_{p}$}

Both the velocity field and the pressure are computed from Stokes equation with correspondent boundary conditions. The velocity of the plunger is used as a parameter to prescribe Dirichlet boundary conditions. It is easy to check that they are proportional to $V_{p}$.

Hence we may consider the velocity and pressure fields at some time $t$ as affine functions of $V_{p}:=V_{p}(t):$

$$
\begin{aligned}
& \mathbf{v}\left(\mathbf{x} ; V_{p}\right)=V_{p} \mathbf{v}(\mathbf{x} ; 1) \\
& p\left(\mathbf{x} ; V_{p}\right)=p_{0}+V_{p}\left(p(\mathbf{x} ; 1)-p_{0}\right) .
\end{aligned}
$$

One can show that this then also holds for the glass force:

$$
F_{g}\left(t ; V_{p}\right)=F_{0}(t)+V_{p}(t)\left(F_{g}(t ; 1)-F_{0}(t)\right),
$$

where $F_{0}(t)$ is the force on the glass as consequence of normal air pressure. By solving the Stokes equations with plunger velocity equal to 1 and tracking the evolution of the free boundary, we can obtain corresponding $\mathbf{v}(\mathbf{x} ; 1), p(\mathbf{x} ; 1)$, and $F_{g}$. As a result of such a process we may e.g. obtain a table with plunger positions, and velocity and pressure fields computed for $V_{p}=1$ in corresponding domains. Hence, the velocity of the plunger can be considered as a function of plunger position, but it is still unknown as a function of $t$.

Let $a(z(t)):=a(z)$ denote the acceleration of the plunger as a function of $z$. From (3.2) it follows

$$
a(t)=a(z)=\frac{V_{p}(z)\left(F_{g}(z ; 1)-F_{0}(z)\right)+F_{0}(z)+F_{p}}{m_{p}},
$$




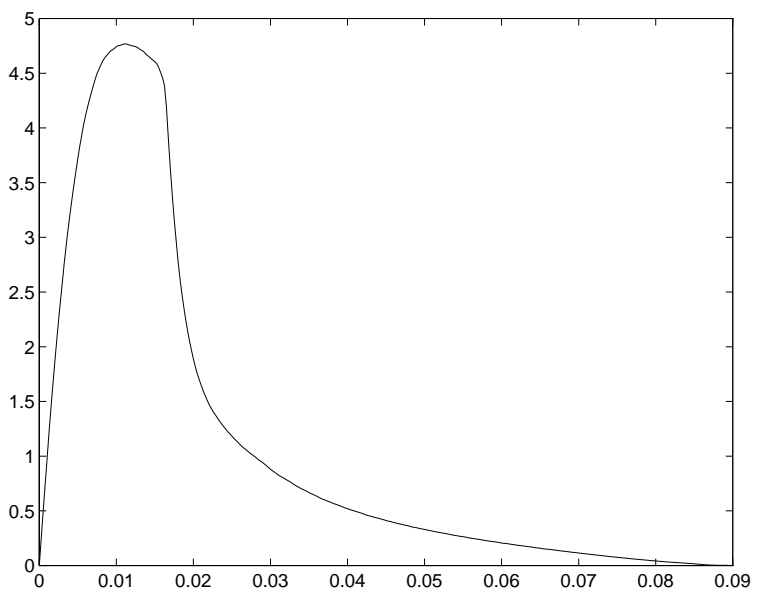

Figure 3.1: Velocity of the plunger as a function of position obtained using implicit scheme.

where $m_{p}$, as was defined before, is the mass of the plunger, and $F_{p}=S_{p} p_{p}=\operatorname{const}(>0)$. Then the basic equation (2.2) we like to solve for the plunger motion, reads

$$
\left\{\begin{aligned}
\frac{1}{2} \frac{d V_{p}^{2}(z)}{d z} & =V_{p}(z) \frac{F_{g}(z ; 1)-P_{0}(z)}{m_{p}}+\frac{F_{p}+P_{0}(z)}{m_{p}}, \\
V_{p}^{0} & =V_{p_{0}}
\end{aligned}\right.
$$

Here we used

$$
\frac{d V_{p}(t)}{d t}=\frac{d V_{p}(z)}{d z} V_{p}(z) .
$$

Now consider an implicit scheme instead. Note that we can implement it now without any explicit predictors for $F_{g}\left(z^{n+1}\right)$ and $V_{p}\left(z^{n+1}\right)$ :

$$
\left\{\begin{aligned}
\frac{1}{2} \frac{V_{p}^{n+1^{2}}-V_{p}^{n 2}}{z^{n+1}-z^{n}} & =\frac{F_{g}\left(z^{n+1} ; 1\right)-P_{0}\left(z^{n+1}\right)}{m_{p}} V_{p}^{n+1}+\frac{F_{p}+P_{0}\left(z^{n+1}\right)}{m_{p}} \\
V_{p}^{0} & =V_{p_{0}} .
\end{aligned}\right.
$$

In Figure 3.1 one can see stability as expected. The velocity of the plunger in Figure 3.1 is a function of $z$. In order to obtain the velocity of the plunger as function of $t$ the following equation may be used

$$
z\left(t^{n+1}\right)=z\left(t^{n}\right)+\Delta t^{n} \quad V_{p}\left(z\left(t^{n}\right)\right) .
$$

The final result is shown in Figure 3.2. 


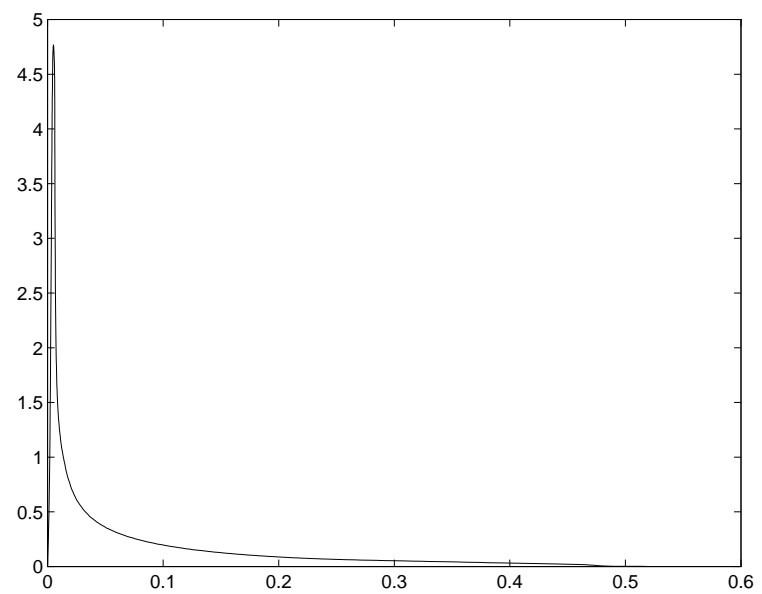

Figure 3.2: Velocity of the plunger as the function of time.

\section{References}

[1] K.Laevsky, R.M.M.Mattheij Mathematical Modeling of Some Glass Problems In Complex Flows in Industrial Processes, Ed. A.Fasano. Birkhäuser 1999.

[2] S.W. Rienstra, T.D. Chandra Analytical Approximations to the Viscous Glass Flow Problem in the Mould-Plunger Pressing Process Including an Investigation of Boundary Conditions to be published 